\title{
All-optically integrated photoacoustic and optical coherence tomography: A review
}

\author{
Wei Qiao and Zhongjiang Chen* \\ Ministry of Education Key Laboratory of Laser Life Science \\ and Institute of Laser Life Science \\ College of Biophotonics \\ South China Normal University \\ Guangzhou 510631, China \\ *chenchj@scnu.edu.cn
}

Received 30 March 2017

Accepted 18 May 2017

Published 27 June 2017

\begin{abstract}
All-optically integrated photoacoustic (PA) and optical coherence tomography (OCT) dualmode imaging technology that could offer comprehensive pathological information for accurate diagnosis in clinic has gradually become a promising imaging technology in the aspect of biomedical imaging during the recent years. This review refers to the technology aspects of alloptical PA detection and system evolution of optically integrated PA and OCT, including Michelson interferometer dual-mode imaging system, Fabry-Perot (FP) interferometer dualmode imaging system and Mach-Zehnder interferometer dual-mode imaging system. It is believed that the optically integrated PA and OCT has great potential applications in biomedical imaging.
\end{abstract}

Keywords: All-optical; photoacoustic; optical coherence tomography; dual-mode imaging.

\section{Introduction}

As a newly noninvasive biomedical imaging technology, photoacoustic imaging (PAI) can specifically detect the optical absorption information of biological tissue, which has rapidly developed in recent years. ${ }^{1,2}$ When the pulsed laser irradiates biological tissue, the absorber within tissue absorbs the light energy to cause local rapid temperature rise, and then temperature rising causes the thermal expansion to produce the pressure wave, called the PA effect. ${ }^{3,4}$ PA signal carrying tissue absorption information is detected by ultrasonic transducer and the image of the absorption structure of the tissue is reconstructed by imaging algorithm. ${ }^{5,6}$ The PAI combines the advantages of high resolution, high contrast of optical imaging and high

*Corresponding author.

This is an Open Access article published by World Scientific Publishing Company. It is distributed under the terms of the Creative Commons Attribution 4.0 (CC-BY) License. Further distribution of this work is permitted, provided the original work is properly cited. 
penetration depth of ultrasound imaging, and avoids the effect of optical scattering on image resolution in principle, enabling deep-range (cm-level) tissue imaging. ${ }^{3,4}$ At the same time, the different components of the tissues have their own special absorption spectrum, which is used for obtaining their distribution. And the number of different components within the tissues and distribution of the tissue are closely related to the pathological characteristics. Therefore, the PAI can not only image the tissue absorption structure information but also reflect the tissue physiological and pathological information. ${ }^{7-13}$ However, there are a large number of weakly absorbed and nonabsorbed tissues in the body. And it is diffcult for PAI to obtain their image information.

Any single imaging technology has its shortcoming, and cannot fully reflect the physiological and pathological information. It is difficult to achieve the exact diagnosis of major diseases, such as cancer, and the efficacy of medication accuracy. ${ }^{14}$ In order to avoid the disadvantage of single imaging technology, it is especially necessary to integrate a variety of imaging techniques to exploit the advantages of various imaging methods. Multimode imaging technology is not a simple combination of various imaging methods, but a variety of imaging equipments and imaging technology integration to complement each other. Therefore, multimode imaging would comprehensively and completely obtain biological cell molecular level, function metabolic levels and anatomical levels of physiological and pathological information. ${ }^{15-18}$

Multiple complementary imaging could offer comprehensive pathological information for accurately diagnosing various diseases in clinic. Thus, there is great interest among researchers in biomedical study to integrate different imaging methods with the PAI system. ${ }^{19-24}$ PA multi-modal imaging technology integrated with OCT, ultrasound imaging and fluorescence have been reported in the literature. ${ }^{25-27} \mathrm{PA}$ multi-modal imaging can simultaneously obtain parameters, such as absorption and scattering of biological tissue, which can provide powerful technical means for modern precision medical diagnosis.

In this review paper, we describe the mechanisms and characteristics of all-optical PA detection technology including Michelson interferometer ${ }^{28-31}$ and FP etalon. ${ }^{32-35}$ Next, we present the current development of multimodality imaging combined with all-optical PAI. At the end, an outlook about all-optical dual-mode imaging system is present.

\section{All-Optical PA Detection}

The PA signal excited by the pulsed laser is a broadband signal that could be fully received by broadband ultrasonic transducer. In addition, the axial resolution of the PAI system is inversely proportional to the bandwidth of ultrasonic transducer. ${ }^{36}$ Therefore, improving the bandwidth of ultrasonic transducer is a key factor for improving the quality of PAI. However, owing to the limitations of piezoelectric materials and fabrication techniques, ultrasonic transducers usually have limited bandwidth. Although the maximum bandwidth of a piezoelectric ultrasonic transducer can reach $120 \%$ of the center frequency, ${ }^{37}$ it is still impossible to achieve a wide range of broadband transducers that can cover from low to high frequencies. Due to the wide band characteristics of optical detectors, it is assumed that optical detection method is used to detect the broadband PA signals achieving high quality PAI.

In recent years, a number of research groups have achieved good results in all-optical PAI. As early as 1990s of the last century, Beard and Mills had begun to use the film FP etalon for the detection of ultrasonic signals. The FP etalon detector has imaging depth of centimeters and a broad bandwidth $(2.3 \mathrm{KPa}$ over $25 \mathrm{MHz}) .{ }^{11}$ This group has achieved intravascular PA signal detection through the coating method in the fiber end integrated FP interferometer. ${ }^{38}$ Chen et al. used polymer micro-ring resonator as a PA signal detection system, the detection bandwidth up to $90 \mathrm{MHz}$, to achieve highresolution imaging of biological tissue. ${ }^{39-41}$ In order to achieve the noncontact detection of ultrasonic signals, Wang Yi et al. employed the low coherence Michelson interferometer (the detection bandwidth up to $17 \mathrm{MHz}$ ) achieving noncontact PA signal detection. ${ }^{28}$ In 2012, Cedric Blatter adopted phasesensitive optical coherence tomography system to realize the noncontact measurements of PA signals (the bandwidth of $13 \mathrm{MHz}$ ), and acquired PA and OCT images of simulated samples. ${ }^{42}$ In the same year, Monchalin et al. utilized the confocal FP interferometer (the axial resolution $20 \mu \mathrm{m}$, the lateral resolution $50-100 \mu \mathrm{m}$ ) for the detection of PA signal and have successfully realized the PA and ultrasonic 
imaging of biological tissue. ${ }^{43}$ In 2014, Seong Jun Park et al. applied an all-fiber heterodyne interferometer (the lateral resolution $1.7 \mu \mathrm{m}$ ) to detect the PA signal and miniaturize the probe. ${ }^{44}$ Here, two representative all-optical PA detection technologies that have been adapted to PAI are Michelson interferometer and FP etalons.

A Michelson interferometer consists of a reference arm and sample arm. ${ }^{28-31}$ The probe is divided into two beams through a beam splitter, one beam is focused through a lens onto a mirror, the other beam is focused on the surface of the sample by a dichroic mirror and an objective lens, and the light is emitted from the sample arm and reflected by the reference arm. Beam is coherent light through the photodetector to receive coherent signal, which formed the Michelson interferometer. The light of the excitation through the dichroic mirror and objective lens was focused on biological tissue. Then, the biological tissue absorbed the light energy to produce the PA signal. The transmission of the PA signal to the surface causes the vibration of the tissue surface, which reflects the PA signal. The intensity $I(t)$ of the low coherence interferometer from the reflective sample surface in the sample arm is given by

$$
\begin{aligned}
I(t) \propto \cos \left(\varphi_{0}(t)\right) & =\csc \left(\varphi_{0}(t)+\varphi_{\mathrm{PA}}(t)\right) \\
& =\cos \left(\frac{2 \pi}{\lambda_{0}}\left(\Delta z_{0}(t)+\Delta z_{\mathrm{PA}}(t)\right)\right),
\end{aligned}
$$

where $\Delta z_{0}(t)$ is the optical path difference between reference and sample arm when the PA excitation laser is off, $\Delta z_{\mathrm{PA}}(t)$ is the PA pressure-induced surface displacement that allows us to reconstruct the distribution of absorbing sample constituents, and $\lambda_{0}$ is the center wavelength of the detection laser. In order to accurately extract the PA signal from the coherence signal, the system has to be working in homodyne mode, i.e., when $\varphi_{0}(t)=k \pi \pm \pi / 2$, the system has the greatest detection sensitivity for acoustic wave. A detailed description of all-optical PA detection mechanism based on Michelson interferometer can be found in Refs. 28-31. One of the great advantages in PA detection based on Michelson interferometer is a broad bandwidth (e.g., $67 \mathrm{MHz}$, as reported by the literature). ${ }^{28-31}$ Another advantage of this method is the noncontact detection. Moreover, the receiving sensitivity of the technology is higher than the conventional transducers.
An FP etalon is another approach for all-optical PA detection. The FP etalon employs an optical interferometer formed by a transparent film sandwiched between a pair of mirrors. ${ }^{32-35}$ The reflected power of the incident laser beam can be modulated by the FP etalon film thickness change. The FP etalon have tens of $\mathrm{MHz}$ detection broadband and size-independent detection sensitivity. A detail description of ultrasound detection mechanism can be found in Ref. 32. The unique merit of the FP etalon, compared with Michelson interferometer, is that the ultrasound detection of the FP interferometer is achieved using a free-space interrogation laser beam. For example, the PA signals can be detected for a two-dimensional (2D) imaging by raster scanning of an interrogation laser beam. The Michelson interferometer and FP etalon PA detection technology integrated with OCT has been applied for multimodality and multi-parameter imaging.

\section{All-Optical PA and OCT Dual-Mode Imaging}

Traditional ultrasonic transducers have to be in contact with the detection object and are not conductive to some cases, such as burning, skin diseases and the affected or ulceration areas. The all-optical PA detection technique can realize noncontact detection of PA, the development of multi-modal imaging system based on all-optical PAI has been favored by more and more researchers. PAI mainly

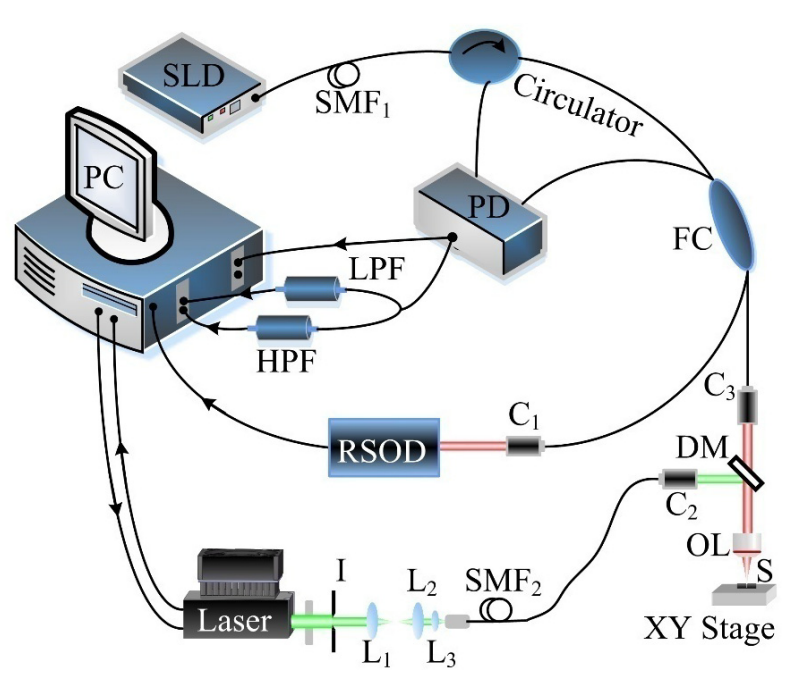

Fig. 1. Schematic representation of the dual-mode simultaneous PA microscopy and optical coherence tomography experimental system. ${ }^{32}$ 


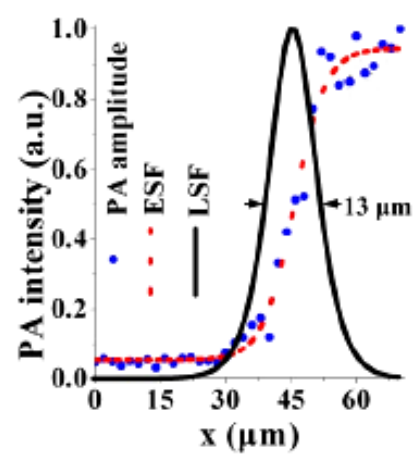

(a)

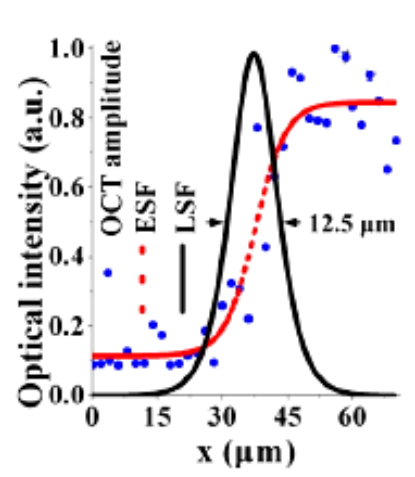

(b)

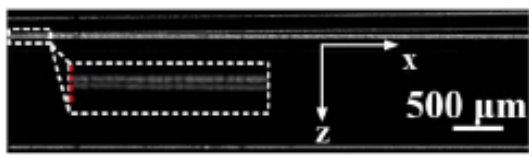

(c)

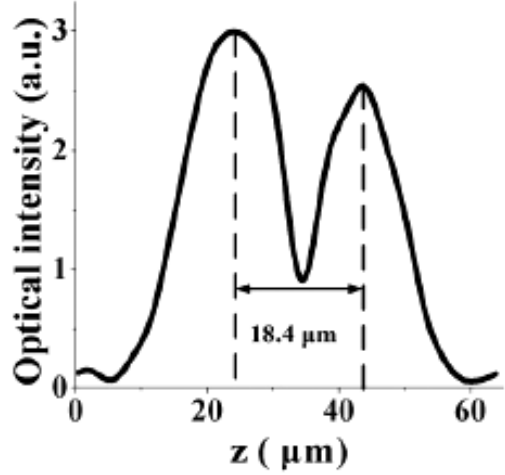

(d)

Fig. 2. Lateral resolution and axial resolution of the dual-mode PAM-OCT imaging system. (a) PAM subsystem. (b) OCT subsystem. (c) OCT B-scan imaging of the air gap between the microscope cover glass and microscope slide. Inset: zoomed image of the white rectangle in $(\mathrm{c})$. (d) The profile along the red dotted line in (c). ${ }^{32}$

relies on the absorption of tissue, but loss of effective information in the low-absorption tissue generally requires external image interpretation. OCT is a relatively mature noninvasive imaging method. ${ }^{45}$ The imaging principle is to detect the coherent light reflected from the sample arm and the reference arm to achieve imaging. The advantage of OCT is sensitivity to the scattering light of tissue, but lacks the absorption information. PAM and OCT dual-mode imaging system can image biological structure and obtain physical parameters simultaneously. ${ }^{46-50}$

\subsection{Michelson interferometer dual-mode imaging system}

The schematic diagram of the dual-mode PAMOCT imaging system based on the Michelson detector is shown in Fig. 1. ${ }^{30}$ The key elements of the co-imaging system are $532 \mathrm{~nm}$ PA excitation source, a Michelson ultrasound sensor for detecting the
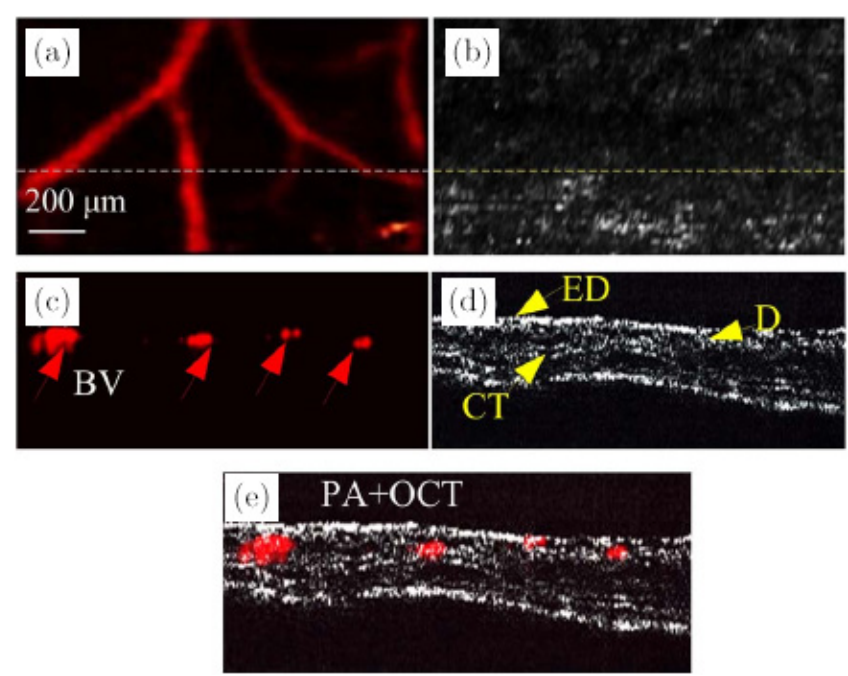

Fig. 3. In vivo simultaneous imaging of a mouse ear by using the dual-mode imaging system. (a) PAM MAP image. (b) OCT MAP image. (c) PAM B-scan image. (d) OCT B-scan image. (e) Fused OCT and PAM images. ED - epidermis; CT cartilage; D — dermis; BV — blood vessel. ${ }^{32}$ 


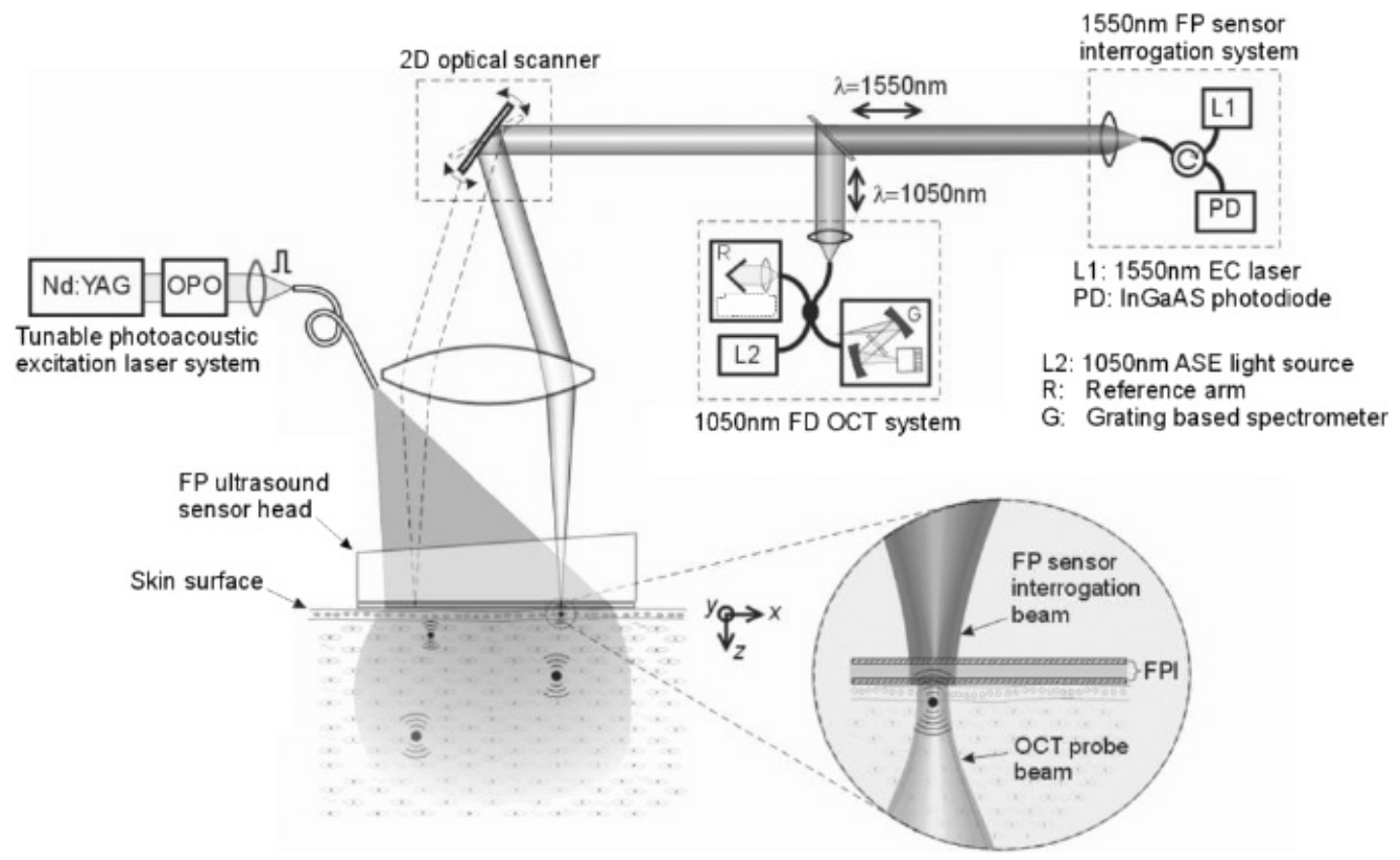

Fig. 4. Multimodal PA-OCT scanner. ${ }^{24}$

PA signals, a $1310 \mathrm{~nm}$ time domain OCT and a photodetector that is used to simultaneously detect the PA and OCT signals. The PAM subsystem contains a PA excitation source and the PA detector based on the Michelson detector. The excitation light (HLX-I-F005, Horus Laser, $532 \mathrm{~nm}$ ) exporting of the fiber tip was the first collimation by a collimator and then focused into the sample by a microscope objective. The Michelson detector consists of a low-coherence light source (central wavelength of $1310 \mathrm{~nm}$ and spectral bandwidth of $45 \mathrm{~nm}$ ) and an all-fiber Michelson interferometer (an optical circulator, a $2 \times 2$ fiber coupler, and a photodetector). The PA signal could be detected by the Michelson detector which resolves the phase difference between reference and sample arm along time, giving access to rapid small path length changes, such as interface displacement due to a transient PA wave. The OCT subsystem at reference arm is achieved by a rapid scanning optical delay line (RSOD), which comprised an optical grating, a Fourier lens, a scanning galvanometer, and a gold-coated reflection mirror. At first, the optical path length of reference arm is equal to the optical path length of the sample arm, the PAM and OCT subsystem starts alternate working, and the

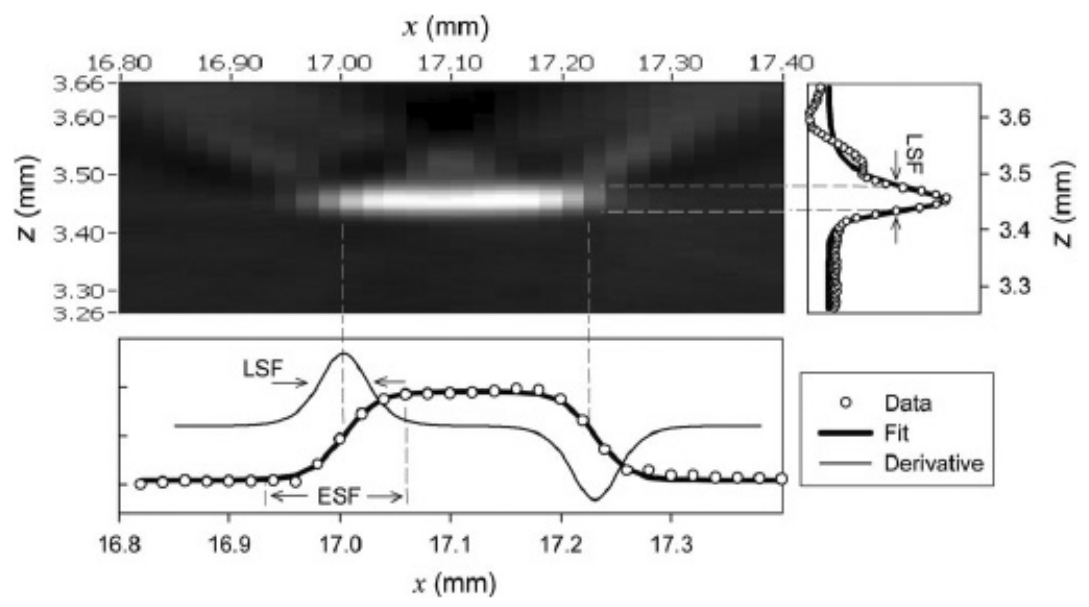

Fig. 5. Estimation of instrument LSF. ${ }^{37}$ 

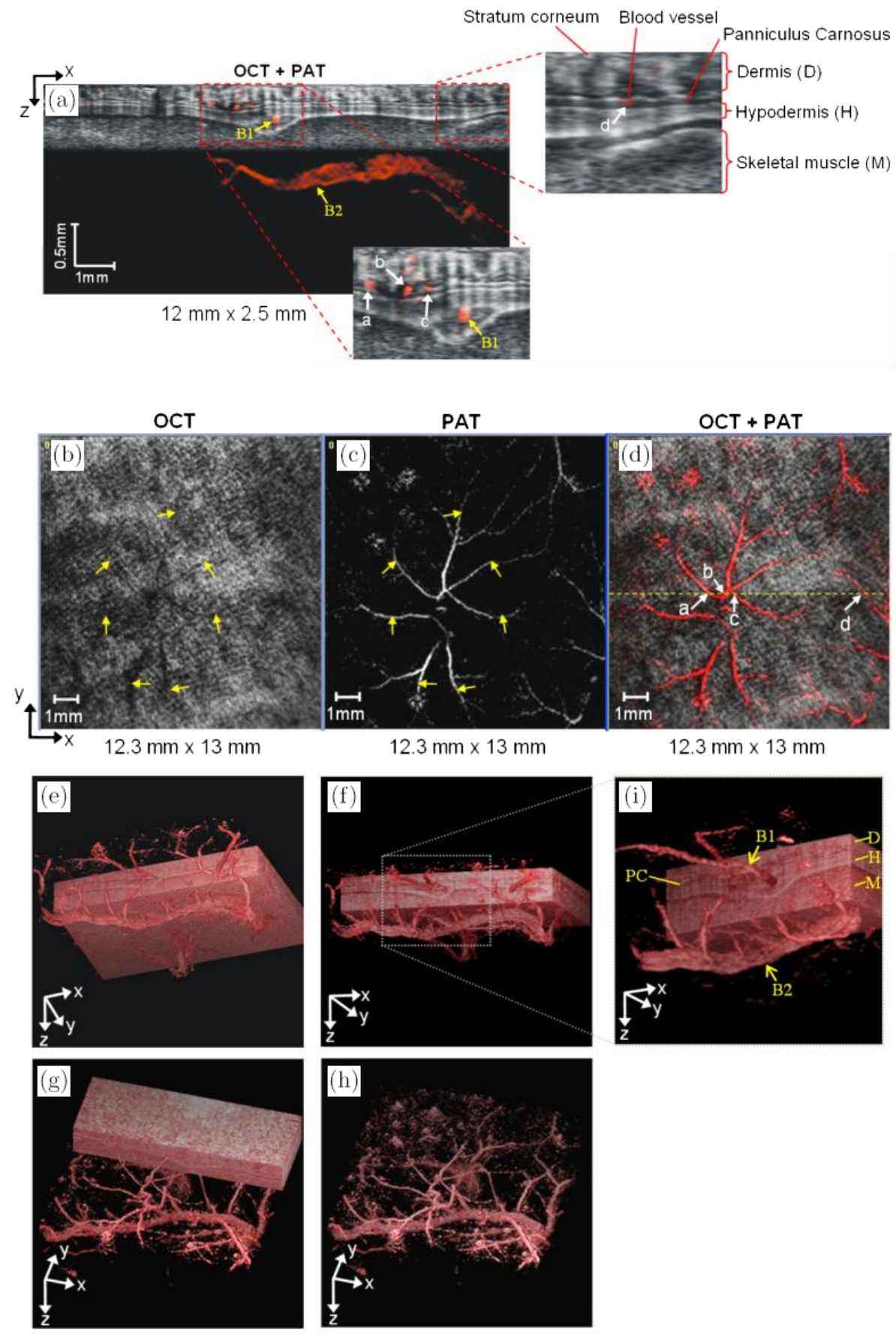

Fig. 6. In vivo OCT and PAT images of the mouse skin. (a) Fused OCT-PAT cross-sectional vertical $(x-z)$ slice. The OCT image shows the layered skin morphology while the PAT image shows several superficial blood vessels within the dermis, Panniculus Carnosus, hypodermis and the skeletal muscle. (b) OCT $(x-y)$ MIP image. (c) PAT $x-y$ MIP image. (d) Fused OCT-PAT $x-y$ MIP image. (e-i) Volume rendered representations of fused OCT-PAM image data at different viewing angles with the OCT image successively resected. ${ }^{24}$

PA and OCT signals are alternately collected by the data acquisition card. The PA and OCT dual-mode imaging based on Michelson interferometer have noncontact detection of higher sensitivity, but the usage of an oil film may limit its applicability. The performance of the dual-mode imaging system is shown in Fig. 2. ${ }^{30}$ The lateral resolution and axial resolution of PAM subsystem were $13 \mu \mathrm{m}$ and $20 \mu \mathrm{m}$, respectively. The lateral resolution and axial resolution of OCT subsystem were $12.5 \mu \mathrm{m}$ and 
$18.4 \mu \mathrm{m}$, respectively. To demonstrate the in vivo microscopic imaging ability of this dual-mode PAM-OCT system, the ear of the mouse was chosen to image in Fig. $3 .^{30}$

\subsection{FP interferometer dual-mode imaging system}

The schematic diagram of the dual-mode PAMOCT imaging system based on the FP detector is shown in Fig. 4. ${ }^{22}$ The key elements of the system are a PA excitation laser system, an FP ultrasound sensor for detecting the PA signals, a $1050 \mathrm{~nm}$ spectral domain OCT (SD-OCT) system and a galvanometer-based optical scan engine that is used to spatially map the PA and OCT signals. The PAT subsystem contains a PA excitation source and the PA detector based on the FP sensor. The PA excitation laser is a fiber-coupled type I optical parametric oscillator pumped by the $355 \mathrm{~nm}$ frequency tripled output of a Q-switched Nd:YAG laser. The divergent beam emerging from the optical fiber is directed on to the FP sensor head, which is acoustically coupled to the skin surface via a drop of water or gel. The incident PA wave is then mapped by raster scanning a focused interrogation laser beam at $1550 \mathrm{~nm}$ over the surface of the sensor using a galvanometer-based $x-y$ scanner. Since the tomographic mode of PA imaging was used in this study, an acoustic back propagation algorithm is required to reconstruct the image from the detected PA signals. A $1050 \mathrm{~nm}$ SD-OCT system was used for OCT image acquisition. $1050 \mathrm{~nm}$ has the advantage of greater penetration depth compared to the spectral range $829-870 \mathrm{~nm}$ used in the previous
PA-OCT dual-mode imaging system. This method has several advantages for combining FP interferometer and OCT. First, the FP sensor will avoid the FOV and SNR limitations of ultrasound receiver. Then the usage of $2 \mathrm{D}$ optical scanning results in a high-speed for PA and OCT imaging. The performance of the dual-mode imaging system is shown in Fig. $5 .{ }^{35}$ The lateral resolution and axial resolution of PAI subsystem were $22 \mu \mathrm{m}$ and $20 \mu \mathrm{m}$, respectively. The axial resolution of the OCT subsystem was $6 \mu \mathrm{m}$. To demonstrate the in vivo imaging ability of this dual-mode PAM-OCT system, the skin of the mouse was chosen to image in Fig. $6 .^{22}$

\subsection{Mach-Zehnder interferometer dual-mode imaging system}

The schematic diagram of the dual-mode PAMOCT imaging system based on the Mach-Zehnder detector is shown in Fig. 7. ${ }^{19}$ A fiber-optic MachZehnder interferometer is applied for PAI. Laser with a wavelength of $1550 \mathrm{~nm}$ from the PAI sample arm is combined with broadband light from the OCT sample arm, separated by a coarse wavelength-division multiplexer (CWDM). The output of CWDM coupled with a collimator was focused to a sample surface by a lens. The reflected light from the sample is collected and sent to CWDM. The two $1310 \mathrm{~nm}$ and $1550 \mathrm{~nm}$ spectral bands are separated and directed back to the respective imaging systems by the CWDM. The PA signal is sampled by an eight-bit digital scope (Le Croy Wave Runner 44XiA); the OCT signal collected by the spectrometer is sent by a computer. A 2D translation stage (Physik Instrumente M-413.22S) is mounted to allow 2D

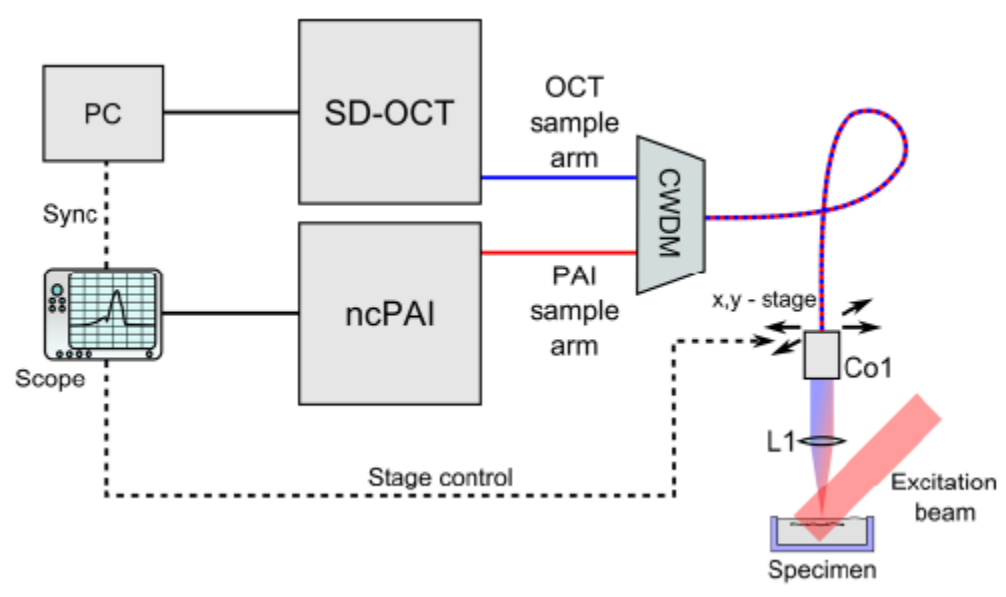

Fig. 7. Multimodal PA-OCT setup. ${ }^{19}$ 


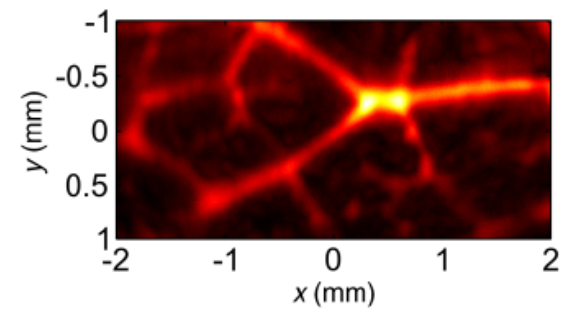

(a)

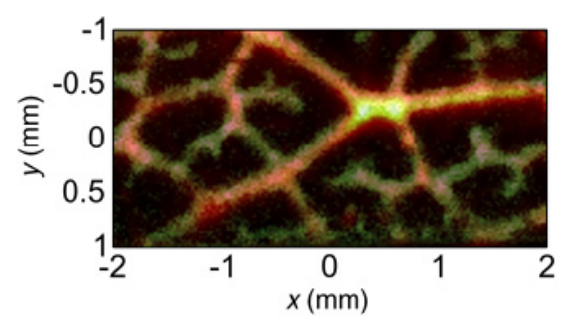

(c)

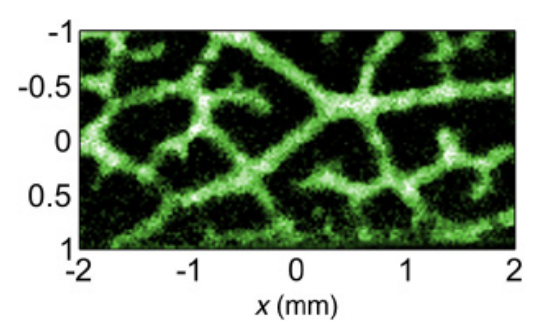

(b)

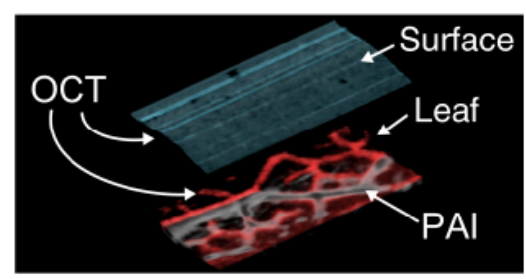

(d)

Fig. 8. Noncontact PAI and OCT measurement of a skeleton leaf/agarose phantom. (a) Maximum intensity plot (MIP) of the PA reconstruction; (b) MIP of the OCT measurement; (c) the overlay of OCT and PAI measurement shows perfect matching; and (d) volume rendering of OCT data and PAI data. ${ }^{19}$

or 3D imaging. The Mach-Zehnder interferometer dual-mode imaging system has its unique advantage (avoiding the usage of oil film) compared to Michelson interferometer dual-mode imaging system. An optical parametric oscillator (OPO, Continuum Surelite OPO Plus) was used to excite ultrasonic waves within the sample. This provides $3-5$ ns pulses over the wavelength region between $689 \mathrm{~nm}$ and $900 \mathrm{~nm}$. To demonstrate the in vivo microscopic imaging ability of this dual-mode PAM-OCT system, the skin of the mouse was chosen to image in Fig. $8 .{ }^{19}$

\section{Outlook and Conclusions}

We have reviewed recent advances in PA and OCT dual-mode imaging system by using all-optical PA detection method. The PAM and OCT dual-mode imaging system simultaneously acquires optical absorption and optical scattering information at high resolution. In general, the absorption and scattering coefficient is an important parameter of the organization because the biological lesions will lead to changes in absorption and scattering coefficient. We can extract the tissue absorption and scattering information to determine the pathological changes. In addition, because of the noncontact detection method, PA-OCT dual-mode imaging system can be easily used for ophthalmology, dermatology and other diseases. The above research results show that the $\mathrm{PA}-\mathrm{OCT}$ dual-mode imaging system can noncontact image the sample and biological tissue with high-resolution imaging, which will have a great potential to the application of dual-mode imaging system in clinical diagnosis.

Single-mode imaging technology cannot comprehensively provide functional and structural information. It is difficult to meet the needs that obtain more abundant information of organisms. All-optical PAI technology has some merits such as high resolution, noncontact, high sensitivity and high imaging depth, which can combine with OCT, ultrasound imaging, fluorescence imaging, second harmonic imaging and so on to provide more information for the future of biomedical diagnosis.

\section{Acknowledgments}

This research is supported by the National Natural Science Foundation of China (61627827; 61331001; 81630046; and 91539127), the Science and Technology Planning Project of Guangdong Province, China (2015B020233016; 2014B020215003; and 2014A020215031), the Distinguished Young Teacher Project in Higher Education of Guangdong, China (YQ2015049), and the Science and Technology Youth Talent for Special Project of Guangdong, China (2015TQ01X882). 


\section{References}

1. L. V. Wang, "Multiscale photoacoustic microscopy and computed tomography," Nat. Photon. 3, 503509 (2009).

2. L. V. Wang, S. Hu, "Photoacoustic tomography: In vivo imaging from organelles to organs," Science 335, 1458-1462 (2012).

3. R. A. Kruger, P. Liu, "Photoacoustic ultrasound: Pulse production and detection in $0.5 \%$ Liposyn," Med. Phys. 21, 1179-1184 (1994).

4. G. Ku, L. V. Wang, "Deeply penetrating photoacoustic tomography in biological tissues enhanced with an optical contrast agent," Opt. Lett. 30, 507509 (2005).

5. X. Wang, Y. Pang, G. Ku et al., "Noninvasive laserinduced photoacoustic tomography for structural and functional in vivo imaging of the brain," Nat. Biotechnol. 21, 803-806 (2003).

6. M. Xu, Y. Xu, L. V. Wang, "Time-domain reconstruction algorithms and numerical simulations for thermoacoustic tomography in various geometries," IEEE Trans. Biomed. Eng. 50, 10861099 (2003).

7. H. F. Zhang, K. Maslov, M. Sivaramakrishnan et al., "Imaging of hemoglobin oxygen saturation variations in single vessels in vivo using photoacoustic microscopy," Appl. Phys. Lett. 90, 053901 (2007).

8. M. Yamazaki, S. Sato, D. Saito et al., "Photoacoustic diagnosis of burns in ratstwo-dimensional photo-acoustic imaging of burned tissue," Biomed. Opt. 4960, 7-13 (2003).

9. E. V. Savateeva, A. A. Karabutov, S. V. Solomatin et al., "Optical properties of blood at various levels of oxygenation studied by time-resolved detection of laser-induced pressure profiles," Int. Symp. Biomed. Opt. 4618, 63-75 (2002).

10. Y. Yao, D. Xing, Y. He et al., "Acousto-optic tomography using amplitude-modulated focused ultrasound and a near-IR laser," Quantum Electron. 31, 1023-1026 (2001).

11. K. Suzuki, Y. Yamohita, K. Ohta et al., "Quantitative measurement of optical parameters in normal breasts using time-resolved spectroscopy: In vivo results of 30 Japanese women," J. Biomed. Opt. 1, 330-334 (1996).

12. X. Wang, Y. Pang, G. Ku et al., "Three-dimensional laser-induced photoacoustic tomography of mouse brain with the skin and skull intact," Opt. Lett. 28, 1739-1741 (2003).

13. A. P. Jathoul, J. Laufer, O. Ogunlade et al., "Deep in vivo photoacoustic imaging of mammalian tissues using a tyrosinase-based genetic reporter," Nat. Photon. 9, 239-242 (2015).
14. M. A. Pysz, S. S. Gambhir, J. K. Willmann, "Molecular imaging: Current status and emerging strategies," Clin. Radiol. 65, 500-516 (2010).

15. J. Tian, J. Bai, X. P. Yan et al., "Multimodality molecular imaging," IEEE Eng. Med. Biol. Magaz. 27, 48-57 (2008).

16. Y. Shi, H. Qin, S. Yang et al., "Thermally confined shell coating amplifies the photoacoustic conversion efficiency of nanoprobes," Nano Res. 9, 3644-3655 (2016).

17. Z. Chen, S. Yang, D. Xing, "In vivo detection of hemoglobin oxygen saturation and carboxyhemoglobin saturation with multiwavelength photoacoustic microscopy," Opt. Lett. 37, 3414-3416 (2012).

18. D. Lee, C. Lee, S. Kim et al., "In vivo near infrared virtual intraoperative surgical photoacoustic optical coherence tomography," Scien. Rep. 6, 35176 (2016).

19. T. Berer, E. Leiss-Holzinger, A. Hochreiner et al., "Multimodal noncontact photoacoustic and optical coherence tomography imaging using wavelengthdivision multiplexing," J. Biomed. Opt. 20, 046013046013 (2015).

20. L. Li, K. Maslov, G. Ku et al., "Three-dimensional combined photoacoustic and optical coherence microscopy for in vivo microcirculation studies," Opt. Exp. 17, 16450-16455 (2009).

21. S. Jiao, Z. Xie, H. F. Zhang et al., "Simultaneous multimodal imaging with integrated photoacoustic microscopy and optical coherence tomography," Opt. Lett. 34, 2961-2963 (2009).

22. E. Z. Zhang, B. Povazay, J. Laufer et al., "Multimodal photoacoustic and optical coherence tomography scanner using an all optical detection scheme for 3D morphological skin imaging," Biomed. Opt. Exp. 2, 2202-2215 (2011).

23. C. Lee, S. Han, S. Kim et al., "Combined photoacoustic and optical coherence tomography using a single near-infrared supercontinuum laser source," Appl. Opt. 52, 1824-1828 (2013).

24. T. Liu, Q. Wei, J. Wang et al., "Combined photoacoustic microscopy and optical coherence tomography can measure metabolic rate of oxygen," Biomed. Opt. Exp. 2, 1359-1365 (2011).

25. Z. Ding, H. Ren, Y. Zhao et al., "High-resolution optical coherence tomography over a large depth range with an axicon lens," Opt. Lett. 27, 243-245 (2002).

26. R. F. Wagner, S. W. Smith, J. M. Sandrik et al., "Statistics of speckle in ultrasound B-scans," IEEE Trans. Sonics Ultrasonics 30, 156-163 (1983).

27. D. R. Larson, W. R. Zipfel, R. M. Williams et al., "Water-soluble quantum dots for multiphoton 
fluorescence imaging in vivo," Science 300, 14341436 (2003).

28. Y. Wang, C. Li, R. K. Wang, "Noncontact photoacoustic imaging achieved by using a low-coherence interferometer as the acoustic detector," Opt. Lett. 36, 3975-3977 (2011).

29. Z. Chen, S. Yang, Y. Wang et al., "Noncontact broadband all-optical photoacoustic microscopy based on a low-coherence interferometer," Appl. Phys. Lett. 106, 043701 (2015).

30. Z. Chen, S. Yang, Y. Wang et al., "All-optically integrated photo-acoustic microscopy and optical coherence tomography based on a single Michelson detector," Opt. Lett. 40, 2838-2841 (2015).

31. Z. Chen, S. Yang, D. Xing, "Optically integrated trimodality imaging system: Combined all-optical photoacoustic microscopy, optical coherence tomography, and fluorescence imaging," Opt. Lett. 41, 1636-1639 (2016).

32. E. Zhang, J. Laufer, P. Beard, "Backward-mode multiwavelength photoacoustic scanner using a planar Fabry-Perot polymer film ultrasound sensor for high-resolution three-dimensional imaging of biological tissues," Appl. Opt. 47, 561-577 (2008).

33. S. Ashkenazi, Y. Hou, T. Buma et al., "Optoacoustic imaging using thin polymer etalon," Appl. Phys. Lett. 86, 134102 (2005).

34. Y. Hou, J. S. Kim, S. Ashkenazi et al., "Broadband all-optical ultrasound transducers," Appl. Phys. Lett. 91, 073507 (2007).

35. P. Morris, A. Hurrell, A. Shaw et al., "A FabryPérot fiber-optic ultrasonic hydrophone for the simultaneous measurement of temperature and acoustic pressure," J. Acoust. Soci. Am. 125, 36113622 (2009).

36. R. A. Noble, A. D. R. Jones, T. J. Robertson et al., "Novel, wide bandwidth, micromachined ultrasonic transducers," IEEE Trans. Ultrasonics Ferroelectrics Freq. Contr. 48, 1495-1507 (2001).

37. K. A. Snook, J. Z. Zhao, C. F. F. Alves et al., "Design, fabrication, and evaluation of high frequency, single-element transducers incorporating different materials," IEEE Trans. Ultrasonics Ferroelectrics Freq. Contr. 49, 169-176 (2002).

38. P. C. Beard, T. N. Mills, "Extrinsic optical-fiber ultrasound sensor using a thin polymer film as a lowfinesse Fabry-Perot interferometer," Appl. Opt. 35, 663-675 (1996).
39. S. W. Huang, S. L. Chen, T. Ling et al., "Low-noise wideband ultrasound detection using polymer microring resonators," Appl. Phys. Lett. 92, 193509 (2008).

40. C. Zhang, T. Ling, S. L. Chen et al., "Ultrabroad bandwidth and highly sensitive optical ultrasonic detector for photoacoustic imaging," ACS Photon. 1, 1093-1098 (2014).

41. B. Dong, S. Chen, Z. Zhang et al., "Photoacoustic probe using a microring resonator ultrasonic sensor for endoscopic applications," Opt. Lett. 39, 43724375 (2014).

42. C. Blatter, B. Grajciar, P. Zou et al., "Intrasweep phase-sensitive optical coherence tomography for noncontact optical photoacoustic imaging," Opt. Lett. 37, 4368-4370 (2012).

43. G. Rousseau, A. Blouin, J. P. Monchalin, "Noncontact photoacoustic tomography and ultrasonography for tissue imaging," Biomed. Opt. Exp. 3, 1625 (2012).

44. S. J. Park, J. Eom, Y. H. Kim et al., "Noncontact photoacoustic imaging based on all-fiber heterodyne interferometer," Opt. Lett. 39, 4903-4906 (2014).

45. Y. Jia, J. C. Morrison, J. Tokayer et al., "Quantitative OCT angiography of optic nerve head blood flow," Biomed. Opt. Exp. 3, 3127-3137 (2012).

46. B. Y. Hsieh, S. L. Chen, T. Ling et al., "All-optical scanhead for ultrasound and photoacoustic imagingImaging mode switching by dichroic filtering," Photoacoustics 2, 39-46 (2014).

47. S. L. Chen, Z. Xie, L. J. Guo et al., "A fiber-optic system for dual-modality photoacoustic microscopy and confocal fluorescence microscopy using miniature components," Photoacoustics 1, 30-35 (2013).

48. B. Dong, H. Li, Z. Zhang et al., "Isometric multimodal photoacoustic microscopy based on optically transparent micro-ring ultrasonic detection," Optica 2, 169-176 (2015).

49. E. Z. Zhang, J. Laufer, B. Povazay et al., "Multimodal simultaneous photoacoustic tomography, optical resolution microscopy, and OCT system," SPIE 7564, 75640U-1 (2010).

50. M. Liu, N. Schmitner, M. G. Sandrian et al., "In vivo three dimensional dual wavelength photoacoustic tomography imaging of the far red fluorescent protein E2-Crimson expressed in adult zebrafish," Biomed. Opt. Exp. 4, 1846-1855 (2013). 